\section{Specific Ionization produced by Positrons and Electrons}

AtтHоUя⿱㇒ ionization produced by an electron in its passage through various gases has been studied by previous workers ${ }^{1}$, to our knowledge no experiment has been performed with the positron which will enable the specific ionization produced by a positron and an electron of the same energy to be compared. In the present experiment, this has been done by investigating the specific ionization by positrons and electrons in nitrogen and argon, using a fully automatic Wilson chamber. Rhodium-102 prepared by bombarding rhodium-103 by fast neutrons was used as the source.

The accompanying table gives the number and length of the tracks studied.

\begin{tabular}{|c|c|c|c|c|}
\hline \multirow[b]{2}{*}{ Llement } & \multicolumn{2}{|c|}{ No. of tracks } & \multicolumn{2}{|c|}{ Length of tracks $(\mathrm{cm})}$. \\
\hline & Positron & Electron & Positron & Electron \\
\hline $\begin{array}{l}\text { Nitrogen } \\
\text { Argon }\end{array}$ & $\begin{array}{l}174 \\
187\end{array}$ & $\begin{array}{l}263 \\
282\end{array}$ & $\begin{array}{l}1,652 \cdot 9 \\
2,169 \cdot 2\end{array}$ & $\begin{array}{l}3,183 \cdot 2 \\
3,257 \cdot 6\end{array}$ \\
\hline
\end{tabular}

Only those positrons and electrons the energy of which lies between $0.18 \mathrm{MeV}$. and $0.96 \mathrm{MeV}$. were taken into account.

Figs. 1 and 2 show the variation with $H_{\rho}$ of specific ionization in nitrogen and argon, respectively, where $H$ is the magnetic field and $\rho$ is the radius of curvature of the track. In the case of nitrogen, the theoretical curve as calculated from the formula of Williams ${ }^{2}$, having regard only to the $2 p$-electrons, is also given. The ionization potential of the $2 p$. electron for nitrogen in the molecular state is not known; consequently, we have taken the value for the atomic state, which is $15.5 \mathrm{eV}$. It can be seen from Fig. 1 that, within experimental error, there is no difference between the specific ionization produced by positrons and that by electrons, and also that the agreement between the theory and experimental results is satisfactory. According to Fig. 2, however, it appears that the specific ionization in argon by positrons is somewhat less than by electrons. It should be noted that at higher energies, where the accuracy of measurement is greater, the divergence between the two values nearly disappears. The difference is noticeable towards the lower energies where the higher concentration of ions along the

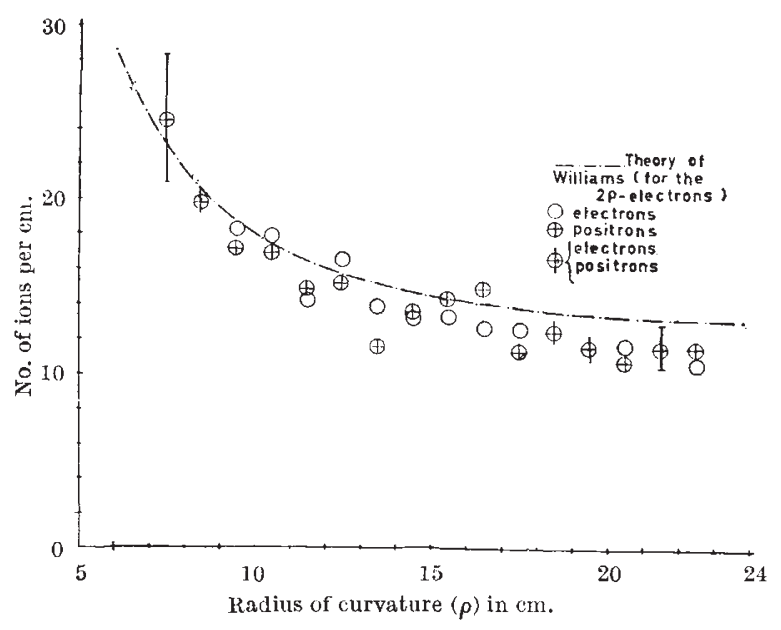

Fig. 1. Variation of primary specific ionization produced by positrons and electrons in nitrogen. $I_{\rho}=204 \times \rho$ gauss-cm.

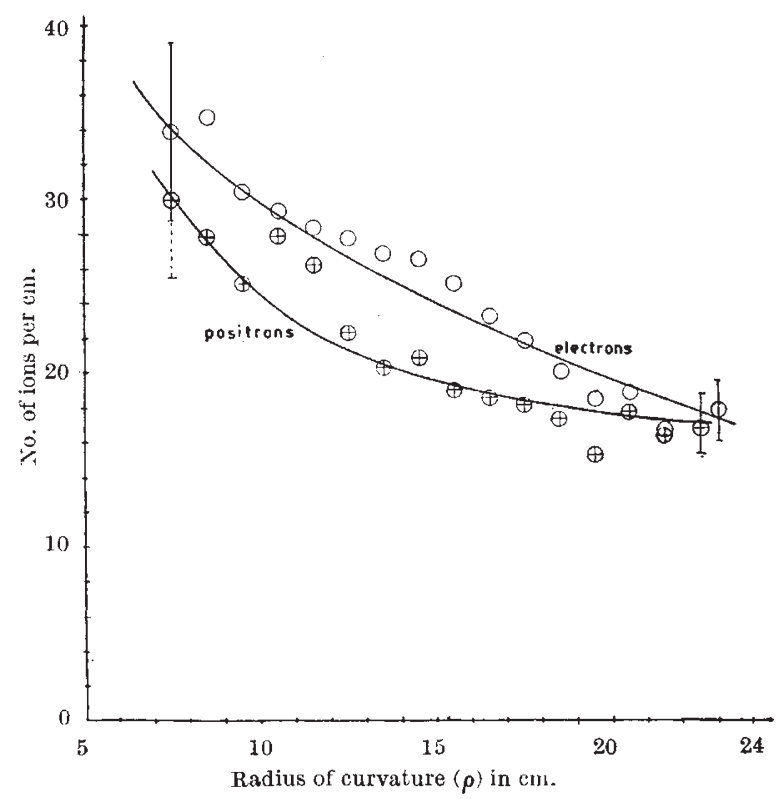

Fig. 2. Variation of primary speciflc ionization produced by positrons and electrons in argon. $I I_{\rho}=204 \times \rho$ gauss $-\mathrm{cm}$.

tracks makes counting them an extremely tedious task, with the result that the subjective error is greater, as it is difficult to resolve two closely spaced ions. For this reason it is not possible to say with certainty whether the difference observed in argon between the positrons and electrons is real. The ideal conditions in which to study this effect would be by employing a low-pressure cloud chamber where greater accuracy in counting the number of ions could be achieved because of the smaller density of ions along the track.

Laboratoire de Physique,

R. R. RoY

M. ETIENNE

Université Libre de Bruxelles, Bruxelles. July 21. 1 Williams and Terroux, Proc. Roy. Soc., A, 126, 289 (1930). Skramstad
and Loughridge, Phys. Rev., 50, 677 (1936).

${ }^{2}$ Williams, Proc. Roy. Soc., A, 139, 163 (1933).

\section{Recombination Coefficient in the $F$ - Regions : a Possible New Process of lonization of Nitrogen Molecules}

Ir is now generally recognized ${ }^{1,2}$ that the high day-time rate of disappearance of electrons in the $E$ - and $F$-regions of the ionosphere cannot be fully explained by the negative-ion formation theory of Bates and Massey ${ }^{3}$, which takes account of the electron loss mainly due to attachment. The high value of the recombination coefficient in the $E$-region and in the upper parts of the $D$-region has recently been explained by A. P. Mitra and Jones ${ }^{4}$ on the basis of dissociative recombination of electrons with $\mathrm{O}_{2}{ }^{+}$and $\mathrm{NO}^{+}$ions, which are believed to be the principal ionized constituents at the heights of these regions. It may therefore be inquired if the process of dissociative recombination may not also be operative in the high $F$-regions $\left(F_{1}\right.$ and $\left.F_{2}\right)$ and be the determining factor for the disappearance of electrons. 